\title{
Thermal and hydrothermal stability of ZrMCM-41 mesoporous molecular sieves obtained by microwave irradiation
}

\author{
T S JIANG*, Y H LI, X P ZHOU, Q ZHAO and H B YIN \\ School of Chemistry and Chemical Engineering, Jiangsu University, Zhenjiang, \\ Jiangsu Province 212013, PR China \\ e-mail: tshjiang@ujs.edu.cn
}

MS received 22 December 2008; revised 19 May 2009; accepted 29 July 2009

\begin{abstract}
ZrMCM-41 mesoporous molecular sieves were synthesized by using the zirconium sulfate as zirconium source and using cetyltrimethyl ammonium bromide as a template under microwave irradiation condition. The samples were characterized by X-ray diffraction (XRD), transmission electron microscopy (TEM), X-ray photoelectron spectroscopy (XPS), inductive coupled plasma (ICP) technique, Fourier transform infrared spectroscopy (FT-IR) and $\mathrm{N}_{2}$ physical adsorption, respectively. The effect of the different initial $\mathrm{ZrO}_{2}: \mathrm{SiO}_{2}$ molar ratio, the different thermal treatment temperature and hydrothermal treatment time on textural property was investigated. The results show that the obtained products possess a typical mesoporous structure of MCM-41 and have specific surface areas in the range of $598 \cdot 1 \sim 971.4 \mathrm{~m}^{2} / \mathrm{g}$ and average pore sizes in the range of ca. $2 \cdot 46 \sim 3.43 \mathrm{~nm}$. On the other hand, the BET specific surface area and pore volume of the synthesized ZrMCM-41 mesoporous molecular sieve decrease with the increased amount of zirconium incorporated in the starting material, the rise of thermal treatment temperature and the prolonging of hydrothermal treatment time, the mesoporous ordering deteriorates. The mesoporous structure of the ZrMCM-41 mesoporous molecular sieve still retains after calcination at $750^{\circ} \mathrm{C}$ for $3 \mathrm{~h}$ or hydrothermal treatment at $100^{\circ} \mathrm{C}$ for 6 days, however, the mesoporous ordering is poor.
\end{abstract}

Keywords. ZrMCM-41 mesoporous molecular sieve; microwave irradiation; thermal and hydrothermal stability; synthesis; characterization.

\section{Introduction}

As a member of M41S family, MCM-41mesoporous molecular sieve with unique pore structure and high specific surface area (about $1000 \mathrm{~m}^{2} / \mathrm{g}$ ) has attracted wide interest among researchers. ${ }^{1}$ Therefore, it has some special applications in the fields of catalysis, adsorption, sensor and petrochemical industry. ${ }^{2-5}$ However, the application of the pure silica MCM-41 mesoporous molecular sieve was limited in petroleum processing industry because of its poor thermal and hydrothermal stabilities, low catalytic activity and weak surface acidity. Recently, many efforts have been made worldwide by researchers for the modification of MCM-41 mesoporous molecular sieve. Some transition metal ions such as $\mathrm{Fe}, \mathrm{Nd}$, $\mathrm{Cu}, \mathrm{Mo},{ }^{6} \mathrm{Co}^{7}$ and $\mathrm{Ni}^{8}$ and so on have been introduced into the framework of MCM-41 mesoporous molecular sieve and these prepared mesoporous

*For correspondence materials have remarkable catalytic performance. The previous literatures reported that a lot of the mesoporous molecular sieves were synthesized by traditional hydrothermal method. However, the hydrothermal synthesis process needs a long crystallization time and high crystallization temperature. Compared with conditional hydrothermal method, microwave irradiation technique was widely applied to the synthesis of mesoporous molecular sieve due to its advantages such as microwave dielectric heating, molecular selective heating, homogeneously and momentarily elevates the temperature of integral synthesis system to crystallization temperature, ${ }^{9-11}$ but most efforts aimed at synthesizing pure silica MCM-41 mesoporous molecular sieve. Investigation on MCM-41 mesoporous molecular sieve with transition metal ions synthesized under microwave irradiation condition was seldom systematically reported.

For this purpose, in the present work, we report here that the synthesis of ZrMCM-41 mesoporous 
Table 1. Material proportion (molar ratio) of the $\mathrm{ZrMCM}-41\left(X^{*}\right)$ samples and $\mathrm{Zr}$ content in the samples.

\begin{tabular}{lcc}
\hline Samples & $n\left(\mathrm{ZrO}_{2}\right): n\left(\mathrm{SiO}_{2}\right): n(\mathrm{CTAB}): n\left(\mathrm{H}_{2} \mathrm{O}\right)$ & $\mathrm{Zr}$ content (wt.\%) \\
\hline ZrMCM-41(a) & $0 \cdot 025: 1: 0 \cdot 2: 70$ & $3 \cdot 16$ \\
ZrMCM-41(b) & $0 \cdot 05: 1: 0 \cdot 2: 70$ & $5 \cdot 58$ \\
ZrMCM-41(c) & $0 \cdot 10: 1: 0 \cdot 2: 70$ & $9 \cdot 59$ \\
ZrMCM-41(d) & $0 \cdot 15: 1: 0 \cdot 2: 70$ & $13 \cdot 40$ \\
ZrMCM-41(e) & $0 \cdot 20: 1: 0 \cdot 2: 70$ & $17 \cdot 51$ \\
ZrMCM-41(f) & $0 \cdot 25: 1: 0 \cdot 2: 70$ & $20 \cdot 07$ \\
ZrMCM-41(g) & $0 \cdot 30: 1: 0 \cdot 2: 70$ & $23 \cdot 17$ \\
\hline
\end{tabular}

$* X=\mathrm{a}, \mathrm{b}, \mathrm{c}, \mathrm{d}, \mathrm{e}, \mathrm{f}$ and $\mathrm{g}$, respectively; $n$-molar.

molecular sieve was carried out by microwave irradiation method. The relationship of the amount of zirconium incorporated and the specific surface area and pore size of the synthesized mesoporous molecular sieve was also investigated. In particular, we focus on the study of the thermal and hydrothermal stabilities of ZrMCM-41 mesoporous molecular sieve. At the same time, the physicochemical properties of the samples were also characterized by a combination of various physicochemical techniques, such as XRD, TEM, FT-IR, XPS, ICP and $\mathrm{N}_{2}$ physical adsorption technique.

\section{Experimental}

\subsection{Synthesis of Zr-containing mesoporous molecular sieve}

Chemicals used in the present work, such as sodium silicate $\left(\mathrm{Na}_{2} \mathrm{SiO}_{3} \cdot 9 \mathrm{H}_{2} \mathrm{O}\right)$, zirconium sulfate $\left(\mathrm{Zr}\left(\mathrm{SO}_{4}\right)_{2}\right.$. $4 \mathrm{H}_{2} \mathrm{O}$ ), cetyltrimethyl ammonium bromide (CTAB), and concentrated sulfuric acid $\left(\mathrm{H}_{2} \mathrm{SO}_{4}\right)$, all analytical reagent grade were purchased from Shanghai Chemical Reagent Corporation, China.

The synthesis of $\mathrm{Zr}$-containing mesoporous molecular sieve was carried out by the microwave irradiation method. The molar ratio of $\mathrm{ZrO}_{2}: \mathrm{SiO}_{2}$ : CTAB : $\mathrm{H}_{2} \mathrm{O}$ in the starting materials was $n$ $(n=0.025,0.05,0 \cdot 1,0 \cdot 15,0 \cdot 2,0.25$ and 0.3 , respectively.): $0 \cdot 2: 1: 70$. A typical synthesis procedure was described as follows: first, a given amount of $\mathrm{Zr}\left(\mathrm{SO}_{4}\right)_{2} \cdot 4 \mathrm{H}_{2} \mathrm{O}$ was dispersed into $30 \mathrm{ml}$ distilled water, $28.42 \mathrm{~g}$ of sodium silicate $\left(\mathrm{Na}_{2} \mathrm{SiO}_{3} \cdot 9 \mathrm{H}_{2} \mathrm{O}\right)$ was dispersed into $50 \mathrm{ml}$ distilled water, and $7.29 \mathrm{~g}$ of CTAB was dispersed into $46 \mathrm{ml}$ distilled water until a gelatinous solution was obtained, and then sodium silicate $\left(\mathrm{Na}_{2} \mathrm{SiO}_{3} \cdot 9 \mathrm{H}_{2} \mathrm{O}\right)$ solution was added into zirconium sulfate solution under stirring. The resulting mixture was slowly added into the gelatinous solution under vigorous stirring for $30 \mathrm{~min}$ and the $\mathrm{pH}$ value of the mixed solution was adjusted to 11 by drop-wise addition of sulfuric acid $(5 \mathrm{~mol} / \mathrm{l})$. After stirring for $80 \mathrm{~min}$ again, the resulting suspension was transferred into a $250 \mathrm{ml}$ round-bottomed flask, and then the round-bottomed flask was placed into a microwave oven with a refluxing condenser and heated at boiling point for $2.5 \mathrm{~h}$ under continuous microwave irradiation with a power of $220 \mathrm{~W}$ (National NN-S570MFS). After cooling to room temperature, the sample was filtered, washed with deionized water, and dried at $120^{\circ} \mathrm{C}$ for $24 \mathrm{~h}$ to obtain a dried sample [denoted as s-ZrMCM-41(X), $X$ stands for the sample number (table 1)]. The s-ZrMCM-41 $(X)$ sample was heated to $550^{\circ} \mathrm{C}$ at a heating rate of $2^{\circ} \mathrm{C} / \mathrm{min}$ and calcined at $550^{\circ} \mathrm{C}$ for $10 \mathrm{~h}$, the calcined sample was designated as $\mathrm{ZrMCM}-41(X)$, and the $\mathrm{Zr}$ content in the sample was determined by the inductive coupled plasma (ICP) is summarized in table 1.

For comparison, the synthesis of the $\mathrm{ZrO}_{2}$ was also carried out via hydrothermal method using zirconium sulfate as zirconium source. The synthesis procedure was described as follows: $7.11 \mathrm{~g}$ of $\mathrm{Zr}\left(\mathrm{SO}_{4}\right)_{2} \cdot 4 \mathrm{H}_{2} \mathrm{O}$ was dispersed into $200 \mathrm{ml}$ distilled water to obtain a $0.1 \mathrm{~mol} / 1$ of zirconium sulfate solution, and then the resultant solution was transferred into the Teflon-lined stainless-steel autoclave and crystallized at $180^{\circ} \mathrm{C}$ for $24 \mathrm{~h}$ in an oven. After cooling to room temperature, the sample was filtered to obtain a white deposition, which was washed three times with deionized water and anhydrous ethanol, respectively. After that, the white deposition was dried at $100^{\circ} \mathrm{C}$ for $24 \mathrm{~h}$ to obtain a dried and white solid $\mathrm{ZrO}_{2}$ sample.

\subsection{Thermal and hydrothermal stability tests}

For thermal stability test, the sample $\mathrm{ZrMCM}-41(X)$ was calcined at 650,750 and $800^{\circ} \mathrm{C}$ for $3 \mathrm{~h}$, 
respectively, denoted as $\mathrm{ZrMCM}-41(X)-650$, ZrMCM-41 $(X)-750$ and ZrMCM-41 $(X)-800$, correspondingly. For hydrothermal stability test, $0.5 \mathrm{~g}$ of ZrMCM-41 $(X)$ was added into a $100 \mathrm{ml}$ Teflon-lined stainless autoclave containing $80 \mathrm{ml}$ of $\mathrm{H}_{2} \mathrm{O}$ and hydrothermally treated at $100^{\circ} \mathrm{C}$ for 2,4 and 6 days, respectively. The hydrothermally treated samples were designated as ZrMCM-41(X)-2d, ZrMCM$41(X)-4 \mathrm{~d}$ and ZrMCM-41(X)-6d, correspondingly.

\subsection{Characterization}

2.3a XRD analysis: The X-ray diffraction (XRD) patterns of samples were recorded with a powder XRD instrument (Rigaku D/max 2500PC) with $\mathrm{CuK}_{\alpha}$ radiation $(\lambda=0 \cdot 15418 \mathrm{~nm})$. The measurement conditions of XRD at low angle are: $40 \mathrm{kV}$, $50 \mathrm{~mA}$, the scanning range is $1-10^{\circ}$ and the scanning speed $1 \% / \mathrm{min}$; the measurement conditions of $\mathrm{XRD}$ at high angle are: $40 \mathrm{kV}, 200 \mathrm{~mA}$, the scanning range is $15-80^{\circ}$ and the scanning speed $4 \% \mathrm{~min}$.

2.3b FT-IR analysis: Fourier transform infrared spectra of samples were recorded on a Nexus FT-IR 470 spectrometer made by Nicolet Corporation (USA) with $\mathrm{KBr}$ pellet technique. The effective range was from 400 to $4000 \mathrm{~cm}^{-1}$.

2.3c Specific surface area and pore size distribution: Specific surface area and pore size were measured by using a NOVA2000e analytical system made by Quntachrome Corporation (USA). The specific surface area was calculated by BrunauerEmmett-Teller (BET) method. Pore size distribution and pore volume were calculated by Barrett-JoynerHalenda (BJH) method. ${ }^{12}$ (see supplementary information table $\mathrm{S} 1$ ).

2.3d TEM images: Transmission electron microscopy (TEM) morphologies of samples were observed on a Philips TEMCNAI-12 with an acceleration voltage of $100-120 \mathrm{kV}$.

2.3e XPS analysis: X-ray photoelectron spectroscopy (XPS) analysis was carried out on an ESCALAB 250 (Thermal Electron Corp.) spectrometer equipped with $\mathrm{Al} \mathrm{K} \alpha \mathrm{X}$-ray source, operating at $150 \mathrm{~W}$. The binding energies were referenced to the $\mathrm{C} 1 \mathrm{~s}$ binding energy at $284.8 \mathrm{eV}$.

2.3f Zirconium content test: The zirconium contents of the synthesized samples were determined by inductive coupled plasma (ICP) (Vista-MAX, Varian).

\section{Results and discussion}

\subsection{XRD analysis}

The powder XRD patterns of mesoporous materials usually exhibit the diffraction peaks in the low angle region. Figure 1 presents the XRD low-angle patterns of the synthesized ZrMCM-41 samples with different $\mathrm{Zr}$ contents after calcination at $550^{\circ} \mathrm{C}$. As shown in figure 1, the ZrMCM-41 (a), ZrMCM-41 (b), ZrMCM-41 (c), ZrMCM-41 (d), ZrMCM-41 (e) and ZrMCM-41 (f) samples, respectively, possess a diffraction peak (100) at $2 \theta$ value of ca. $2 \cdot 3^{\circ}$, and has small and weak diffraction peaks $(110,200$ and 210 , respectively) at $2 \theta$ value ranging from $4^{\circ}$ to $6^{\circ}$, which is consistent with the characteristic diffraction pattern of the typical MCM-41 mesoporous molecular sieve, ${ }^{1}$ indicating that the synthesized samples have typical hexagonal mesoporous structure of MCM-41. A small amount of zirconium ions incorporated into the framework of the MCM-41 mesoporous molecular sieve does not significantly modify the crystalline structure. However, when the amount of $\mathrm{Zr}$ is added in the starting materials

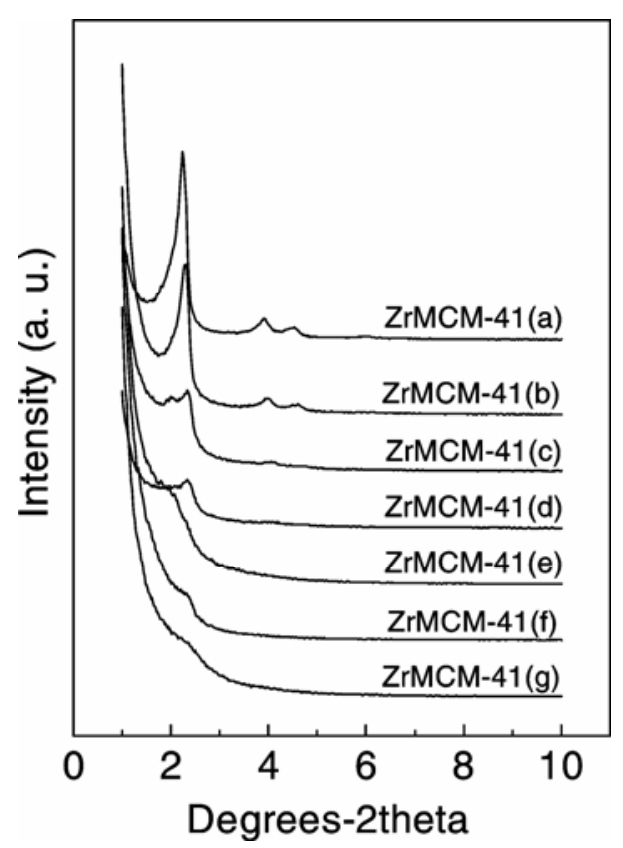

Figure 1. Small angle XRD patterns of the samples synthesized according to the different molar ratios of $\mathrm{ZrO}_{2}: \mathrm{SiO}_{2}: \mathrm{CTAB}: \mathrm{H}_{2} \mathrm{O}$. The all samples were calcined at $550^{\circ} \mathrm{C}$ for $10 \mathrm{~h}$, respectively. 
$0.3 \mathrm{~mol}$, the diffraction peak (100) of the ZrMCM41 (g) sample can not be obvious observed from figure 1 , indicating the mesoporous ordering of the ZrMCM-41 sample is poor. Additionally, from figure 1 , the diffraction peak (100) of the calcined ZrMCM-41 sample was broad and weak with the increase of the amount of zirconium ions incorporated into MCM-41 framework, and the intensity of the diffraction peak (100) also decreased, showing that the long-range ordering of the ZrMCM-41 samples synthesized under microwave irradiation condition decreased with the increase of the amount of zirconium incorporated, which agrees with the results from the literature. ${ }^{13}$

The XRD high-angle patterns of the ZrMCM-41 (c) calcined at $550^{\circ} \mathrm{C}$ and the $\mathrm{ZrO}_{2}$ samples are shown in figure 2 , respectively. As shown in figure 2, the characteristic peaks of the bulk $\mathrm{ZrO}_{2}$ phase were not observed in the XRD pattern of the calcined ZrMCM-41 (c) sample, certifying that the framework of MCM-41 mesoporous molecular sieve still retained amorphous when zirconium ions were incorporated into the framework of the ZrMCM-41 (c) sample, zirconium incorporated into the inner and outer channel of MCM-41 mesoporous molecular sieve did not gather to $\mathrm{ZrO}_{2}$ clusters, and highly dispersed into the amorphous wall of MCM-41, indicating that $\mathrm{Zr}$ ions might have been incorporated into the mesoporous framework. ${ }^{14}$

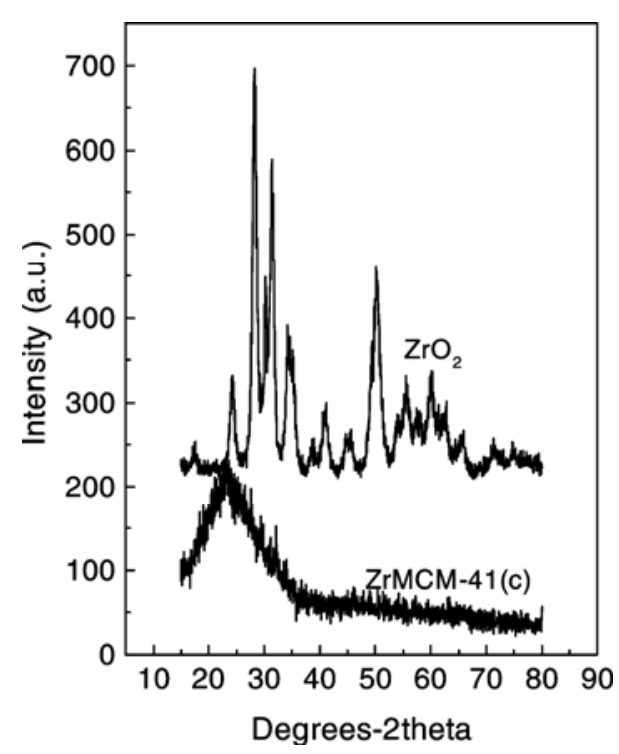

Figure 2. Wide angle XRD patterns of the ZrMCM41(c) synthesized according to the molar ratio of $0 \cdot 1: 1: 0 \cdot 2: 70\left(\mathrm{ZrO}_{2}: \mathrm{SiO}_{2}:\right.$ CTAB $\left.: \mathrm{H}_{2} \mathrm{O}\right)$ and the $\mathrm{ZrO}_{2}$ synthesized via hydrothermal method.

\section{$3.2 \quad F T-I R$ analysis}

The FT-IR spectra of the synthesized samples with different zirconium contents after calcination at $550^{\circ} \mathrm{C}$ show that the band at $3500 \mathrm{~cm}^{-1}$ is characteristic of the adsorbed water molecules; the bands at $1620-1640 \mathrm{~cm}^{-1}$ are due to the vibration of the adsorbed water molecules; the band about $810 \mathrm{~cm}^{-1}$ is the symmetric stretching vibration band of $\mathrm{Si}-\mathrm{O}_{-}$ $\mathrm{Si}$; the band about $460 \mathrm{~cm}^{-1}$ is assigned to rocking vibration of $\mathrm{Si}-\mathrm{O}-\mathrm{Si}$. The band indicating $\mathrm{Si}-\mathrm{O}-\mathrm{Si}$ asymmetric stretching vibration, which is found at $1100 \mathrm{~cm}^{-1}$ for pure silica, the $\mathrm{Si}-\mathrm{O}-\mathrm{Si}$ bands of the ZrMCM-41(b), ZrMCM-41(c), ZrMCM-41(e) and ZrMCM-41(g) samples shifted to 1082, 1074, 1067 and $1052 \mathrm{~cm}^{-1}$, respectively. The shift in stretching frequency is attributed to deteriorating silica framework after incorporation of zirconium ions. ${ }^{15}$ Additionally, from FT-IR spectra, the band at other wavenumber also shifted, indicating that the influence of various vibrations of the framework was caused by incorporating zirconium ions into the framework of MCM-41 mesoporous molecular sieve (see supplementary information figure $\mathrm{S} 1$ ).

As can be observed in FT-IR spectra of the synthesized ZrMCM-41 (c) before and after calcination at $550^{\circ} \mathrm{C}$, the bands at 2921,2850 and $1480 \mathrm{~cm}^{-1}$ are the characteristic bands of the surfactant alkyl chains. After the ZrMCM-41 (c) sample was calcined at $550^{\circ} \mathrm{C}$ for $10 \mathrm{~h}$, the bands at 2921, 2850 and $1480 \mathrm{~cm}^{-1}$ disappeared, certifying that the template has been effectively removed (see supplementary information figure S2).

\subsection{XPS analysis}

Figure 3 shows the XPS spectrum of the ZrMCM-41 (c) sample. As seen in figure 3, the sample is mainly consisted of $\mathrm{O}, \mathrm{Si}, \mathrm{C}, \mathrm{Zr}$ and $\mathrm{Na}$ elements. Of which the $\mathrm{C}$ element is mainly due to the $\mathrm{C}$ absorbed in the air, and the part is probably from the remains of the decomposition of the template CTAB. Na element is from the raw material sodium silicate.

The Ols XPS spectrum of the ZrMCM-41 (c) sample is presented in figure 4. As shown in figure $4, \mathrm{O} 1 \mathrm{~s}$ can be deconvolved into three components, showing three different environments for oxygen, the peak located at $532 \mathrm{eV}$ is probably from the $\mathrm{Si}-$ $\mathrm{OH}$ bond. The peak located at $533 \mathrm{eV}$ can be assigned to the characteristic of the $\mathrm{Si}-\mathrm{O}-\mathrm{Si}$ bond; while the peak located at $530.95 \mathrm{eV}$ is due to the $\mathrm{Si}$ - 
$\mathrm{O}-\mathrm{Zr}$ bond. Additionally, as can be observed from figure 3, the XPS signals of $\mathrm{O} 1 \mathrm{~s}$ are at least $0.75 \mathrm{eV}$ higher than that of $\mathrm{ZrO}_{2}(530 \cdot 2 \mathrm{eV})$, which indicates the presence of framework zirconium. Moreover, the binding energies of $\mathrm{Zr} 3 d_{5 / 2}(183.26 \mathrm{eV})$ are higher than that of $\mathrm{ZrO}_{2}(182 \cdot 2 \mathrm{eV}){ }^{16}$

\subsection{TEM analysis}

The morphological features of the ZrMCM-41 materials calcined at $550^{\circ} \mathrm{C}$ were investigated by TEM. Figure 5 illustrates TEM images of the four calcined samples ZrMCM-41 (b), ZrMCM-41 (c), ZrMCM41 (e) and ZrMCM-41 (g), respectively. As shown in figure 5, the ZrMCM-4l (b), ZrMCM-41 (c) and ZrMCM-41 (e) samples possess a typical hexagonal arrangement mesoporous structure of MCM-41. Combined with XRD high-angle patterns (figure 2), certifying that ZrMCM-41 mesoporous molecular

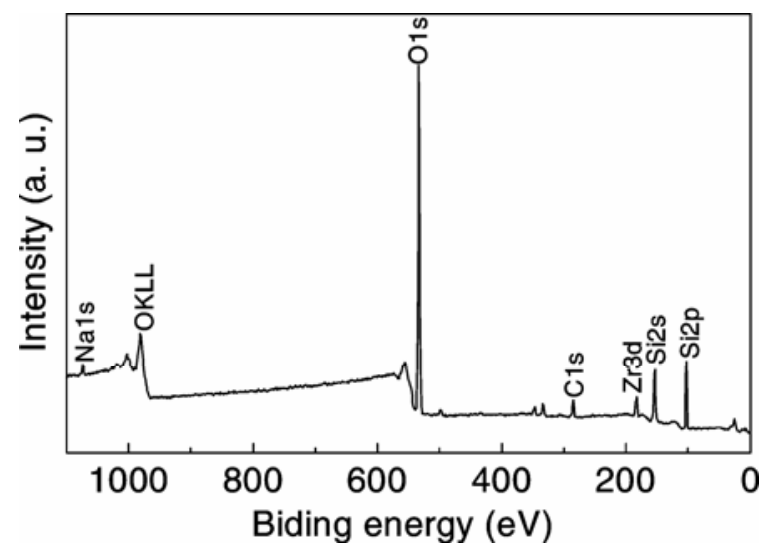

Figure 3. XPS spectrum of the ZrMCM-41(c) sample.

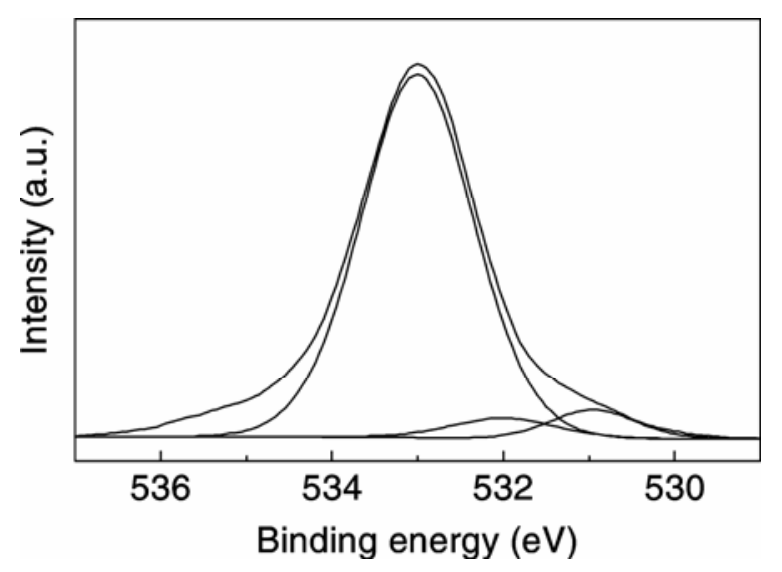

Figure 4. O1s XPS spectrum of the ZrMCM-41(c) sample. sieve was successfully synthesized by microwave irradiation method. Additionally, as can been observed in figure 5, though the ZrMCM-4l(g) sample did not exhibit obvious mesoporous structure, combined with the specific surface area data $\left(598.1 \mathrm{~m}^{2} / \mathrm{g}\right)$ (see supplementary information table $\mathrm{S} 1)$, the ZrMCM-4l(g) sample should have the mesoporous structure with the relatively low mesoporous ordering. This is attributed to too high zirconium content into the mesoporous framework, and the channels in ordered hexagonal array were partly damaged, resulting in the low mesoporous ordering. Therefore, it is reasonable to be concluded that the ordering of the synthesized ZrMCM41 mesoporous material decreased with the increase of zirconium content incorporated into the mesoporous framework of MCM-41. Further, the $\mathrm{ZrO}_{2}$ particles were not observed from figure 5 , indicating that zirconium was incorporated into the framework of mesoporous molecular sieve, which is consistent with the results of FT-IR and XPS analysis.

TEM images of the ZrMCM-41 (c) sample after thermal treatment at $750^{\circ} \mathrm{C}$ for $3 \mathrm{~h}$ or hydrothermal treatment at $100^{\circ} \mathrm{C}$ for 6 days are shown in figure 6 . From figure 6, the ZrMCM-41(c) sample still retained the mesoporous structure after thermal treatment at $750^{\circ} \mathrm{C}$ for $3 \mathrm{~h}$ or hydrothermal treatment at $100^{\circ} \mathrm{C}$ for 6 days. However, compared with the TEM image of the ZrMCM-4l (c) calcined at $550^{\circ} \mathrm{C}$ for $10 \mathrm{~h}$, the mesoporous ordering deteriorated.

\subsection{Nitrogen adsorption}

The $\mathrm{N}_{2}$ adsorption-desorption isotherms and pore size distribution curves of the synthesized samples after calcination at $550^{\circ} \mathrm{C}$ are shown in figure 7 . The $\mathrm{N}_{2}$ adsorption-desorption isotherms of the synthesized ZrMCM-41 (c) sample after thermal treatment at different temperatures $\left(550,650\right.$ and $750^{\circ} \mathrm{C}$, respectively), or hydrothermal treatment at different time (2, 4 and 6 days, respectively) are illustrated in figure 8. From Figure 7A, the isotherms of the ZrMCM-41(b), ZrMCM-41(c) and ZrMCM-41(e) samples exhibit typical type IV isotherms, certifying that the three samples have typical mesoporous structure. ${ }^{1}$ Additionally, the three isotherms exhibit a sharp capillary condensation step at a relative pressure $\left(P / P_{0}\right)$ of ca. $0 \cdot 3-0 \cdot 4$, showing that the three samples have uniform pore size distribution and larger pore volume. Moreover, as shown in figure 7A, 


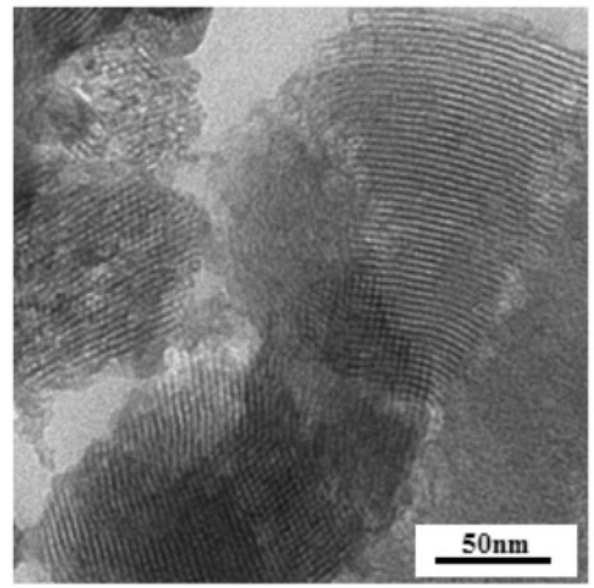

ZrMCM-41(b)

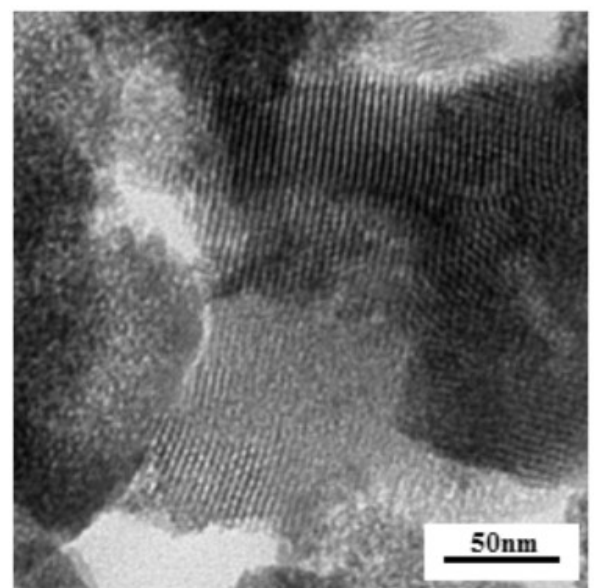

ZrMCM-41(e)

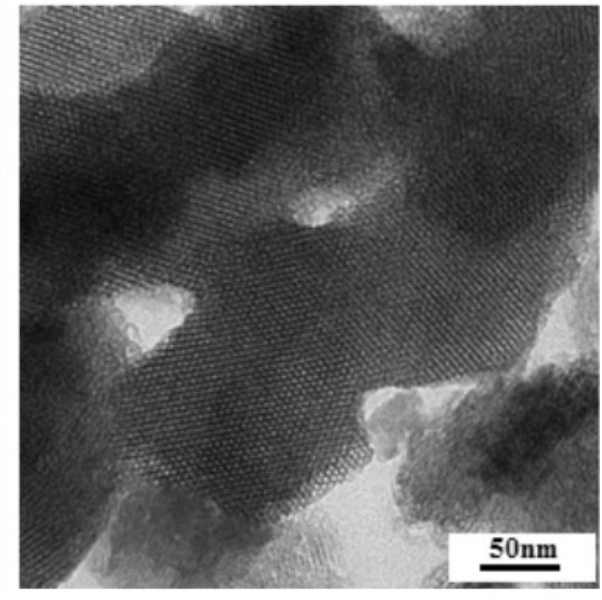

ZrMCM-41(c)

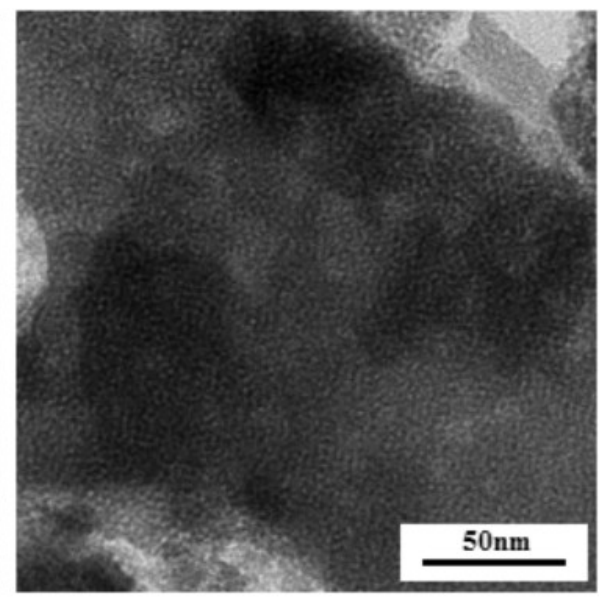

$\mathrm{ZrMCM}-41(\mathrm{~g})$

Figure 5. TEM images of the synthesized samples after calcination at $550^{\circ} \mathrm{C}$.

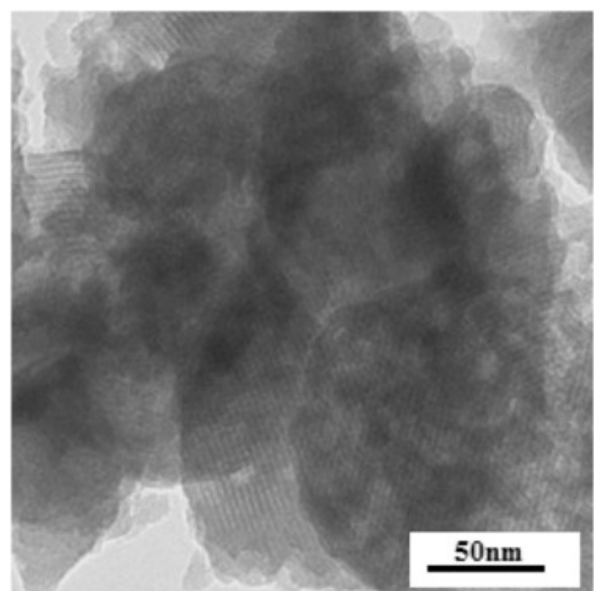

ZrMCM-41(c)-750

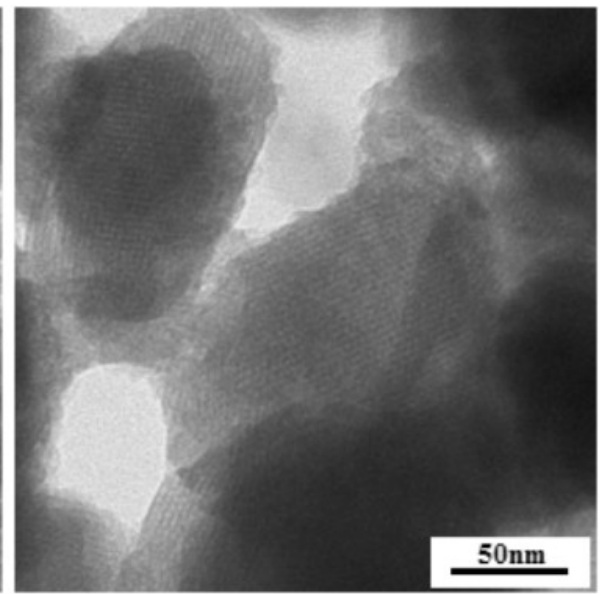

ZrMCM-41(c)-6d

Figure 6. TEM images of the ZrMCM-41(c) sample after thermal treatment at $750^{\circ} \mathrm{C}$ for $3 \mathrm{~h}$ or hydrothermal treatment at $100^{\circ} \mathrm{C}$ for $6 \mathrm{~d}$.

the isotherms of the ZrMCM-41 (g) sample did not belong to typical type IV isotherms, but combined with the surface area data $\left(598.1 \mathrm{~m}^{2} / \mathrm{g}\right)$ (see supple- mentary information table S1), the ZrMCM-41(g) sample still has the mesoporous structure, however, the ordering is poor. On the other hand, from 

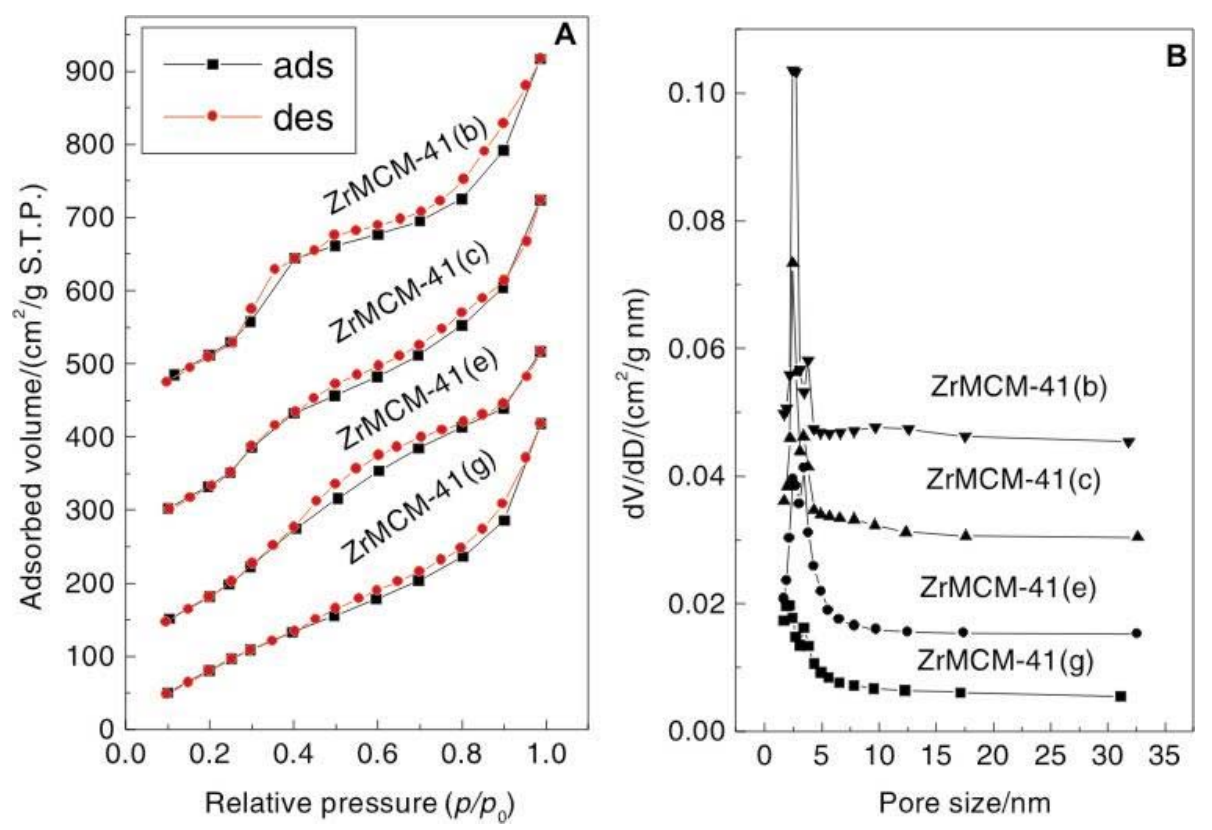

Figure 7. $\mathrm{N}_{2}$ adsorption-desorption isotherms (A) and pore size distribution curves $(\mathbf{B})$ of the synthesized samples after calcination at $550^{\circ} \mathrm{C}$.

figure $7 \mathrm{~A}$, the isotherms of the four samples with hysteresis loop caused by capillary condensation in mesopores, further indicating that they have the mesoporous structure. Compared with the isotherms of other sample, the isotherms of the ZrMCM-4l (b) has a sharper capillary condensation step at the same $p / p_{0}$, illustrating that it possesses more uniform pore size distribution. As can be observed in figure 7B, narrow and sharp peaks can be observed in an average pore size range of $2-3.5 \mathrm{~nm}$ for the ZrMCM41(b), ZrMCM-41(c) and ZrMCM-41(e) samples after calcination at $550^{\circ} \mathrm{C}$, showing that the three samples have uniform pore size distribution as well as. In addition, the pore size distribution curves of the samples were broad and weak as zirconium content in synthesized mesoporous materials increased, which reflects that the increase of zirconium ions incorporated into the silica framework of MCM-41 mesoporous molecular sieve, caused the distortion of the mesoporous structure of MCM-41, resulting in the uneven pore size distribution.

As shown in figure 8A, while the synthesized ZrMCM-41 (c) sample was calcined at $750^{\circ} \mathrm{C}$ for $3 \mathrm{~h}$, the $\mathrm{N}_{2}$ adsorption-desorption isotherms exhibit type IV isotherms, showing that the ZrMCM-41 (c)750 sample still retains the mesoporous structure. On the other hand, from figure $8 \mathrm{~B}$, after the ZrMCM-41 (c) sample was hydrothermally treated at $100^{\circ} \mathrm{C}$ for 6 days, the $\mathrm{N}_{2}$ adsorption-desorption isotherms still belong to type IV isotherms, manifesting that the ZrMCM-41 (c)-6d sample still has the mesoporous framework. Combined with the TEM analysis, the mesoporous ordering of the two samples (ZrMCM-41 (c)-750 and ZrMCM-41 (c)$6 \mathrm{~d}$, respectively) decreased as compared with the ZrMCM-41 (c). From the specific surface area data calculated by BET method (see supplementary information table $\mathrm{S} 1$ ), we can see that after the ZrMCM-41 (c) sample was calcined at $800^{\circ} \mathrm{C}$ for $3 \mathrm{~h}$, the specific surface area of the ZrMCM-41(c)800 sample was much smaller than that of the ZrMCM-41 (c), indicating that the mesoporous structure was mostly damaged. Therefore, it is reasonable to conclude that ZrMCM-41 mesoporous molecular sieve synthesized via microwave irradiation method possesses high thermal and hydrothermal stabilities.

Additionally, from results of $\mathrm{N}_{2}$ the physical adsorption (see supplementary information table $\mathrm{S} 1$ ), the specific surface areas and pore volumes of the synthesized samples after calcination at $550^{\circ} \mathrm{C}$ for $10 \mathrm{~h}$ was decreased as the zirconium content increased. The calcined ZrMCM-41 samples have specific surface areas in the range of $598.1-971.4 \mathrm{~m}^{2} / \mathrm{g}$ and average pore sizes in the range of $2.46-3.43 \mathrm{~nm}$. Combined with the results of analysis of XRD and $\mathrm{N}_{2}$ physical adsorption, it is reasonable to conclude that the mesoporous ordering of the sample 

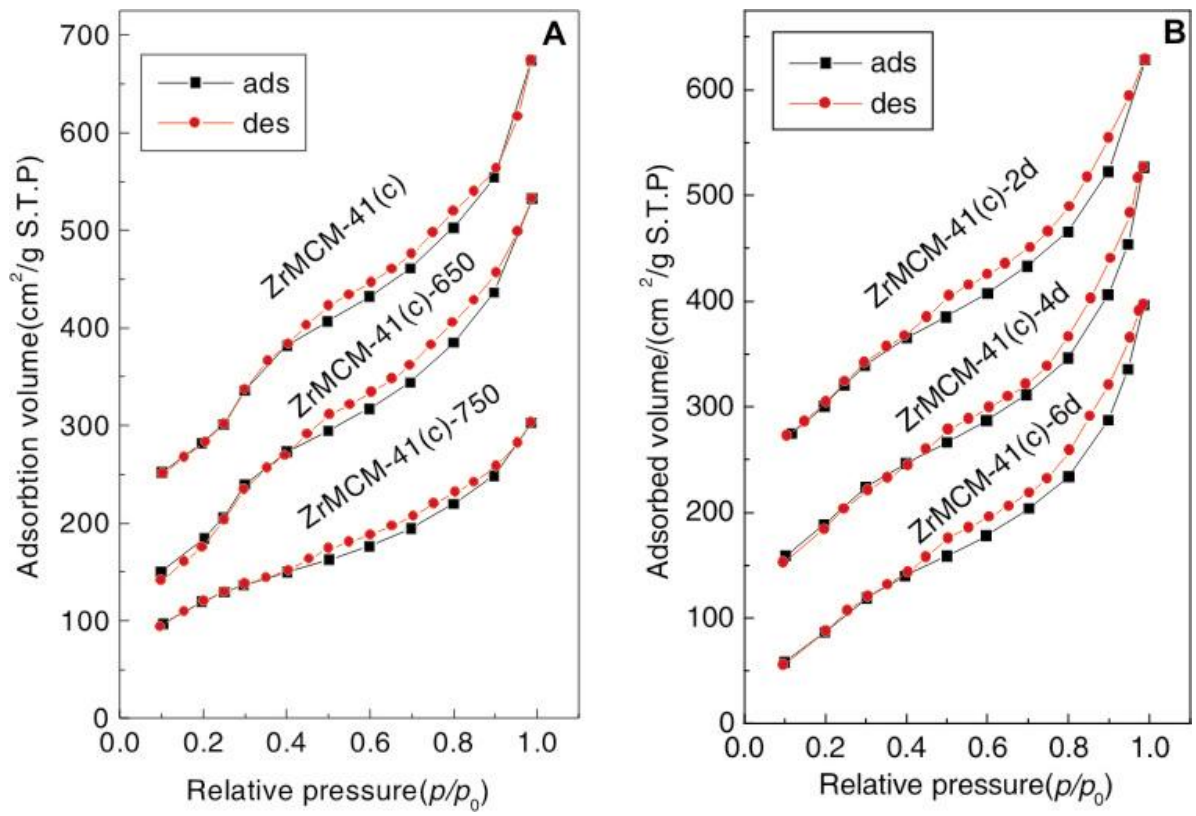

Figure 8. $\mathrm{N}_{2}$ adsorption-desorption isotherms of the ZrMCM-41(c) sample after calcination at different temperatures $\left(550,650\right.$ and $750^{\circ} \mathrm{C}$, respectively) (A) or hydrothermal treatment at different time (2, 4 and 6 days) (B).

deteriorated with the increase of the content of zirconium incorporated into the MCM-41 mesoporous molecular sieve. On the other hand, the pore sizes of the synthesized samples after calcination at $550^{\circ} \mathrm{C}$ for $10 \mathrm{~h}$ gradually increased as the zirconium content increased. These observations show that zirconium incorporation strongly affects the textural properties of the resultant materials. Usually, the pore shrinkage or enlargement of the sample after transition metals incorporation is related to the diameter of the transition metal ions. The pore size of the ZrMCM-41 sample can be increased due to the size of the $\mathrm{Zr}^{4+}$ ion $(r=0.084 \mathrm{~nm})$ is much larger than that of the $\mathrm{Si}^{4+}$ ion $(r=0.026 \mathrm{~nm}),{ }^{17}$ this result suggests that the $\mathrm{Zr}$ ions are indeed inserted into the silica framework. In addition, the specific surface areas and the pore volumes of the samples decreased with the rise of the calcination temperature or the prolonging of hydrothermal treatment time, and the mesoporous ordering deteriorated. The synthesized ZrMCM-41 mesoporous molecular sieves have the specific surface areas in the range of 598.1$971.4 \mathrm{~m}^{2} / \mathrm{g}$, indicating that the ZrMCM-41 mesoporous molecular sieve with high specific surface area can be synthesized via microwave irradiation method in the molar ratio $\left(\mathrm{ZrO}_{2}: \mathrm{SiO}_{2}\right)$ range of $0 \cdot 025-0 \cdot 3$.

\section{Conclusions}

Ordered hexagonal ZrMCM-41 mesoporous molecular sieves with different zirconium contents and high specific surface area were successfully synthesized by microwave irradiation method. After the synthesized sample was calcined at $550^{\circ} \mathrm{C}$ for $10 \mathrm{~h}$, the template was effectively removed. Zirconium incorporation strongly affects the textural properties of the resultant materials. The specific surface area and pore volume of the ZrMCM-41 mesoporous molecular sieve decreased as the zirconium content increased, and the mesoporous ordering deteriorated. On the contrary, the pore size increased. Additionally, the structural regularity of the synthesized ZrMCM41 mesoporous molecular sieve was strongly affected by the calcination temperature and hydrothermal treatment time. The specific surface areas, pore volumes and the mesoporous ordering of the synthesized ZrMCM-41 mesoporous molecular sieves decreased with the rise of calcination temperature and the prolonging of the hydrothermal treatment time, respectively. On the other hand, the mesoporous structure of the ZrMCM-41 mesoporous molecular sieve was still retained after calcination at $750^{\circ} \mathrm{C}$ for $3 \mathrm{~h}$ or hydrothermal treatment at $100^{\circ} \mathrm{C}$ for 6 days. However, the mesoporous ordering deteriorated as 
compared with the ZrMCM-41(c) sample. High zirconium content is unfavourable to the formation of the ZrMCM-41 with highly ordered mesoporous structure. The ZrMCM-41 mesoporous molecular sieve synthesized by microwave irradiation method has high thermal and hydrothermal stabilities.

\section{Acknowledgements}

The authors would like to thank the associated professor Kangmin Chen, the Test Centre of Physics and Chemistry, Jiangsu University for his kind help in characterizing the samples with TEM analysis. Financial support from Industry Key Item Fund of Zhenjiang City (GY2006017) is gratefully acknowledged.

\section{Supplementary information}

The FT-IR spectra of the synthesized samples with different zirconium contents after calcination at $550^{\circ} \mathrm{C}$ are shown in supplementary figure $\mathrm{S} 1$. The FT-IR spectra of the synthesized ZrMCM-41 (c) before and after calcination at $550^{\circ} \mathrm{C}$ are shown in supplementary figure $\mathrm{S} 2$. The specific surface area data calculated by BET method and the pore size distribution and pore volume data calculated by $\mathrm{BJH}$ method are listed in supplementary table S1. For supplementary information see www.ias.ac.in/chemsci (website).

\section{References}

1. Beck J S, Vartuli J C, Roth W J, Leonowicz M E, Kresge C T, Schmitt K D, Chu C T W, Olson D H,
Sheppard E W, McCullen S B, Higgins J B and Schlenker J L 1992 J. Am. Chem. Soc. 11410834

2. Aoyama N, Yoshihara T, Furukawa S I, Nitta T, Takahashi H and Nakano M 2007 Fluid Phase Equilib 257212

3. Jiang T S, Lu L D, Yang X J, Zhao Q, Tao T, Yin H B and Chen K M 2008 J. Porous mater. 1567

4. Kerby M C, Degnan Jr T F, Marler D O and Beck J S 2005 Catal. Today 10455

5. Mary E G and Kenneth J B 1999 Microporous Mesoporous Mater. 28113

6. Ziolek M, Nowak I, Kilos B, Sobczak I, Decyk P, Trejda M and Volta J C 2004 J. Phys. Chem. Solids. $\mathbf{6 5} 571$

7. Zhao Q, Chu J Y, Jiang T S and Yin H B 2007 Colloids Surf. A301 388

8. Jehng J M, Tung W C and Kuo C H 2008 J. Porous Mater. 1543

9. Park S E, Kim D S, Chang J S and Kim W Y 1998 Catal. Today 44301

10. Fantini M C A, Matos J R, Cides da Silva L C, Mercuri L P, Chiereci G O, Celer E B and Jaroniec M 2004 Mater. Sci. Eng. B112 106

11. Hwang Y K, Chang J S, Kwon Y U and Park S E 2004 Microporous Mesoporous Mater. 6821

12. Barrett E P, Joyner L G and Halenda P P $1951 \mathrm{~J}$. Am. Chem. Soc. 73373

13. Wang X X, Lefebvre F, Patarin J and Basset J M 2001 Microporous Mesoporous Mater. 42269

14. Chien Y C, Wang H P, Liu S H, Hsiung T L, Tai H S and Peng C Y 2008 J. Hazard. Mater. 151461

15. Salas P, Wang J A, Armendariz H, Angeles-Chavez C and Chen L F 2009 Mater. Chem. Phys. 114139

16. Jones D J, Jiménez-Jiménez J, Jiménez-López A, Maireles-Torres P, Olivera-Pastor P, RodriguezCastellón E and Rozière J 1997 Chem. Commun. 5 431

17. Chen L F, Wang J A, Noreña L E, Aguilar J, Navarrete J, Salas P, Montoya J A and Ángel P D $2007 \mathrm{~J}$. Solid State Chem. 1802958 